\title{
The Use of Lean Six Sigma Methodology in Increasing Capacity of a Chemical Production Facility at DSM
}

\author{
Marco Meeuwse*
}

\begin{abstract}
Lean Six Sigma is an improvement method, combining Lean, which focuses on removing 'waste' from a process, with Six Sigma, which is a data-driven approach, making use of statistical tools. Traditionally it is used to improve the quality of products (reducing defects), or processes (reducing variability). However, it can also be used as a tool to increase the productivity or capacity of a production plant. The Lean Six Sigma methodology is therefore an important pillar of continuous improvement within DSM. In the example shown here a multistep batch process is improved, by analyzing the duration of the relevant process steps, and optimizing the procedures. Process steps were performed in parallel instead of sequential, and some steps were made shorter. The variability was reduced, giving the opportunity to make a tighter planning, and thereby reducing waiting times. Without any investment in new equipment or technical modifications, the productivity of the plant was improved by more than $20 \%$; only by changing procedures and the programming of the process control system.
\end{abstract}

Keywords: Lean Six Sigma · Chemical Production · Debottlenecking

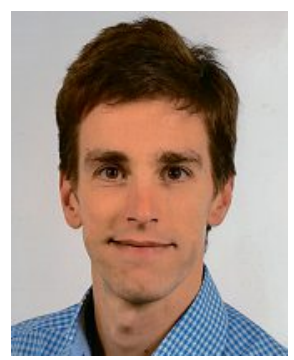

spinning disc reactor'. In his current role he focuses on the improvement of (bio-) chemical production plants, in terms of capacity, yield and costs.

\section{Introduction Lean Six Sigma}

Lean Six Sigma (LSS) is an improvement method, or basis of an improvement culture, combining the principles of Lean and Six Sigma. Lean focusses on removing 'waste' from processes; i.e. taking out all activities in a process which do not generate value. Goal is to keep the process flowing, removing waiting times or large inventories, leading to an agile, effective and efficient process. The concepts of Lean have their basis in the automotive industry, where Toyota has had great success with it. ${ }^{[1]}$

Six Sigma is a method to improve the quality of a process, reducing defects (e.g. in products) and decrease variability. This method stems from the electronics industry, where Motorola developed the concept. ${ }^{[2]}$ Six Sigma is originally a quality tool; the name is derived from the statistical measurement of variance, where a 'Six Sigma' process produces only 3.4 defect products per 1 million items. Statistics play an important role in Six Sigma; improvements need to be proven statistically, otherwise they do not 'count'. Six Sigma focusses on the use of data as the basis for decisions, improvements, etc. The use of statistical tools like Design of Experiments (DoE) and regression analysis to find causal relationships is also an integral part of Six Sigma.
Lean Six Sigma is a combination of both methods; aiming at utilizing the advantages of both worlds. ${ }^{[3]}$ Within DSM it is used to improve the quality of products, but also of processes, e.g. to stabilize the process performance. The method is especially useful to solve problems with a (previously) unknown cause, using brainstorms and statistical investigations. It is also used in projects aimed at increasing the capacity or product output of the manufacturing plants, and is therefore also part of the continuous improvement culture. This paper describes an example of such a project.

Lean Six Sigma projects commonly follow the 'DMAIC' approach, consisting of the five project phases: Define, Measure, Analyze, Improve and Control. In the Define phase the scope of the project is defined, and the primary metric is chosen, which quantifies the goal of the project. The Measure phase determines whether this goal can be measured with sufficient accuracy, to be able to significantly prove the difference made by the project. In the Analyze phase the problem is analyzed and ideas are derived to solve the problem, e.g. using brainstorming, but also statistical analysis of data, experiments, etc. In the Implement phase the prioritized ideas are implemented and their effect is observed on short term. The Control phase verifies over a longer time period whether the primary metric has improved significantly and sustainably; this last phase also shows the benefit of the project, in terms of the metric as well as financially. 


\section{Example of a Debottlenecking Project using LSS}

Lean Six Sigma consists of a large number of tools, describing all of them is out of scope for this article. Instead an example of a project will be given, where several aspects of Lean Six Sigma have been applied. This project was started to increase the capacity ('debottlenecking') of one of the production facilities of DSM Nutritional Products in Grenzach, Germany. The data shown here have been anonymized, in order to protect the process know-how and intellectual property. It demonstrates, however, how Lean Six Sigma has been applied to improve the output of this process plant. The project is presented using the phases of the DMAIC approach.

The process under consideration is a batch process. It consists of two consecutive reactors and a buffer vessel, followed by a centrifugation, a storage silo, a drying step and finally the product is filled in big-bags. The process is schematically depicted in Fig. 1. Parallel to the process a recovery system for remaining product and solvents from the mother liquor is present. In the recovery system several improvements have been made as well, but are not described in this article.

A multidisciplinary team has been assigned to this project. Some of the team members have been working in this plant for a longer time, others came in with a 'fresh' view. Especially the new team members joined the shift teams for several days. Getting to know the plant not only on paper, but also knowing what practical issues the operators encounter, was very helpful for the project. The overall project duration was about half a year.

\section{Define Phase (D)}

The Define phase, also commonly referred to as scoping phase, is meant to align the project team and all the relevant stakeholders, on a clear and common goal. In this phase the business case for the project is defined as well.

The goal of this project was to increase the capacity of this plant in the coming years. For the process it was defined which capacity is needed by when; in total this process should be able to produce over $20 \%$ more than in the past years. It was decided that the project will focus on reducing the average cycle time of the process, instead of optimizing yield or increasing batch size. The cycle time is defined as the time between the start of two consecutive batches. Reducing this cycle time means that more batches per year can be produced, and therefore more product.

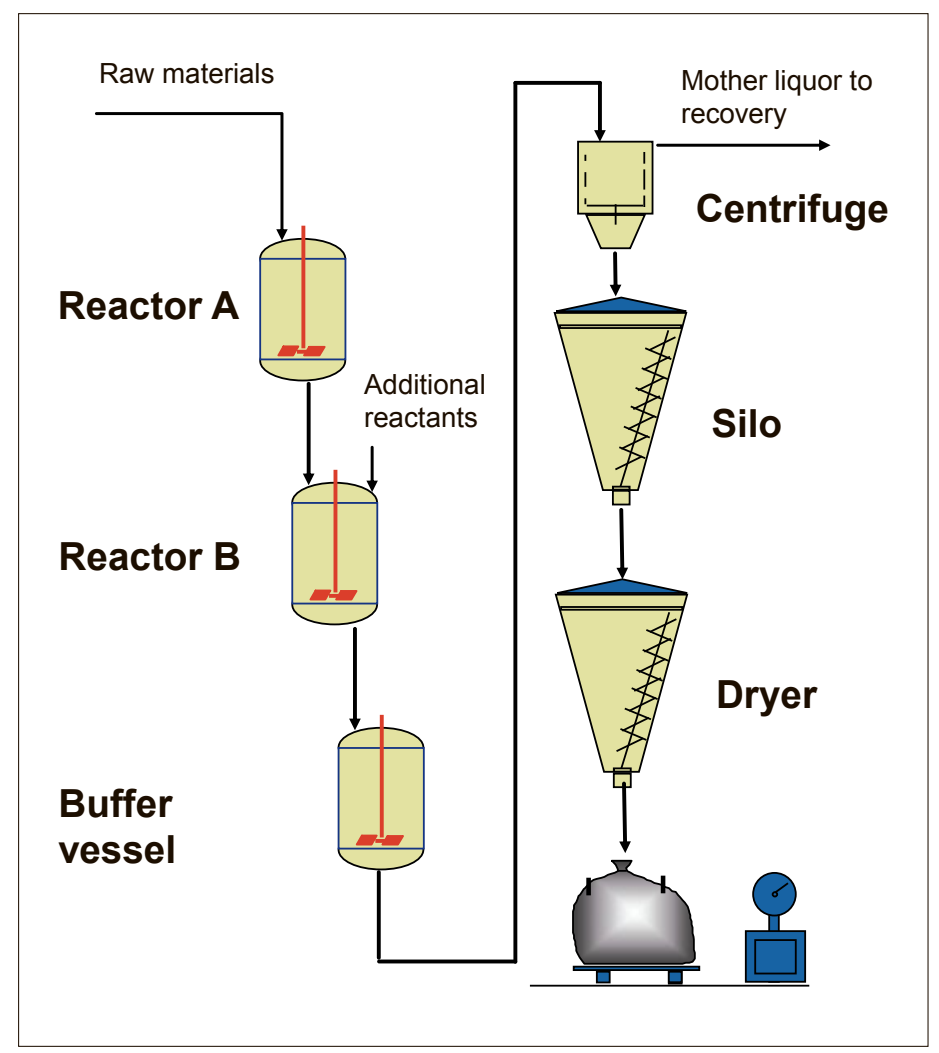

Fig. 1. Schematic flow chart of the process.

\section{Measure Phase (M)}

The goal of the project was to increase the production rate, by increasing the number of batches. Historically, and logically, in the production the cycle time per batch was defined as the time between finalizing the packaging of two consecutive batches. However, the drying/packaging unit was not the unit operation taking the longest time, and therefore not the time-limiting step. In a first analysis it was shown that reactor $\mathrm{B}$ was the unit taking the longest time. It was therefore decided to take the cycle time of this unit as the "primary metric' for this project. This means, the success of the project will be measured using this parameter; at the end of the project it should be shown that the cycle time has been reduced significantly. The duration of the other unit operations will also be analyzed and improved; especially if these are not much smaller than the duration of reactor $\mathrm{B}$, otherwise these will become the bottleneck if reactor $\mathrm{B}$ is improved substantially. Additionally, it also helps if the shorter unit operations are improved, although this may not reduce the overall cycle time or increase daily production capacity (hard benefit); however, the 'soft benefit' of increased flexibility can help minimize production loss in case of technical breakdowns or other process disturbances, and increases the robustness of the process.

During the Measure phase a measurement system analysis has been performed on this primary metric. Goal of this anal- ysis is to prove that the data is reliable enough to draw conclusions on improvements. This is an important step, because it often shows that measurement devices or chemical analysis (including sample preparation) are not precise enough to show the (sometimes small) improvements aimed for. Therefore, a large number of data points or samples will be necessary to prove whether the primary metric has improved; if unlucky, months or even years would be needed for the control phase. This should be checked beforehand, so that the measurement system can be improved, e.g. by using a different analysis method or measurement principle, or by standardizing the measurement procedure. For example, for a chemical analysis (HPLC, GC, ...) a Gauge Repeatability und Reproducibility (Gauge R\&R) can be performed. ${ }^{[3]}$ Here $x$ samples are taken, analyzed y times, by $\mathrm{z}$ different people. The variations in the results show how precise the measurement itself is (variation in results per sample), but also what the effect of the person performing them is (are the procedures clear and consistent) and how that is in relation with the variation expected in the process (e.g. if a concentration in the process varies between 10 and $20 \mathrm{~g} / \mathrm{l}$ an accuracy of $0.01 \mathrm{~g} / \mathrm{l}$ would give no added value).

In Fig. 2 the cycle time of reactor B is shown, for a period of 4 months preceding the start of the improvements. This was taken as the baseline of the analysis, and was also used to compare the results after completion of the project. The cycle time 
data are derived from the process control system, which records every program step for each unit operation. The measurement system analysis showed that the data acquisition frequency at the beginning was not sufficient to capture all process steps. Some process steps were therefore missing, leading to inaccurate times. The settings of the process control system needed to be adjusted, to make the data suitable and accurate. With these adjustments the cycle time was recorded reliably. The resulting cycle times showed an average of 17.2 hours, with a standard deviation of one hour. If this process could be improved by one hour, approximately 30 data points would be needed to prove this improvement as statistically significant with a probability greater than $95 \%$. This would take only 20 days, such an improvement can therefore easily be proven after the project, despite the variation. However, the variability should be decreased anyway, for a successful project.

In Lean Six Sigma it is also common to define a 'secondary metric'; a quantity which should not be influenced negatively due to the project. In this case this is the output per batch, which is a measure for the yield; this should not be decreased after the improvements on the cycle time have been made. For the secondary metric it should also be proven that the data quality is sufficient.

\section{Analyze Phase (A) and Implement Phase (I)}

During the Analyze phase the necessary data is gathered, and analyzed to develop improvement ideas. The result of the analysis phase is a list of ideas, including an indication of chance of success and required effort (work to implement, as well as costs). After the Analyze phase the ideas with most benefit and/or least effort will be implemented during the Implement phase. For clarity, in this article the description of both phases is combined, although they were executed in series, and only the ideas with the most potential and which have been implemented, are described.

The first task was to define the average duration and its variation for every unit operation. A visual representation (box plot) is shown in Fig. 3. It clearly shows that reactor $\mathrm{B}$ and the dryer have a duration much longer than the other process steps. The focus of the process improvement therefore needed to be on these unit operations. The overall cycle time of the process (measured on reactor B) was mostly determined by the duration of the process steps in the reactor; the variation in the drying time, however, was larger, and 'slow' batches in the dryer could therefore also limit the overall cycle time. Both process steps need to be analyzed in more detail in order to in- crease the capacity. The other process steps have also been investigated, although they are not the bottleneck. The improvements in these steps led to increased flexibility; however, these 'soft benefits' are not described in the article.

\section{Reactor $B$}

The overall duration of the process in reactor $\mathrm{B}$ was split into a number of process stages, as shown in Fig. 4. These process stages were defined in such a way that all the relevant steps can be separated,

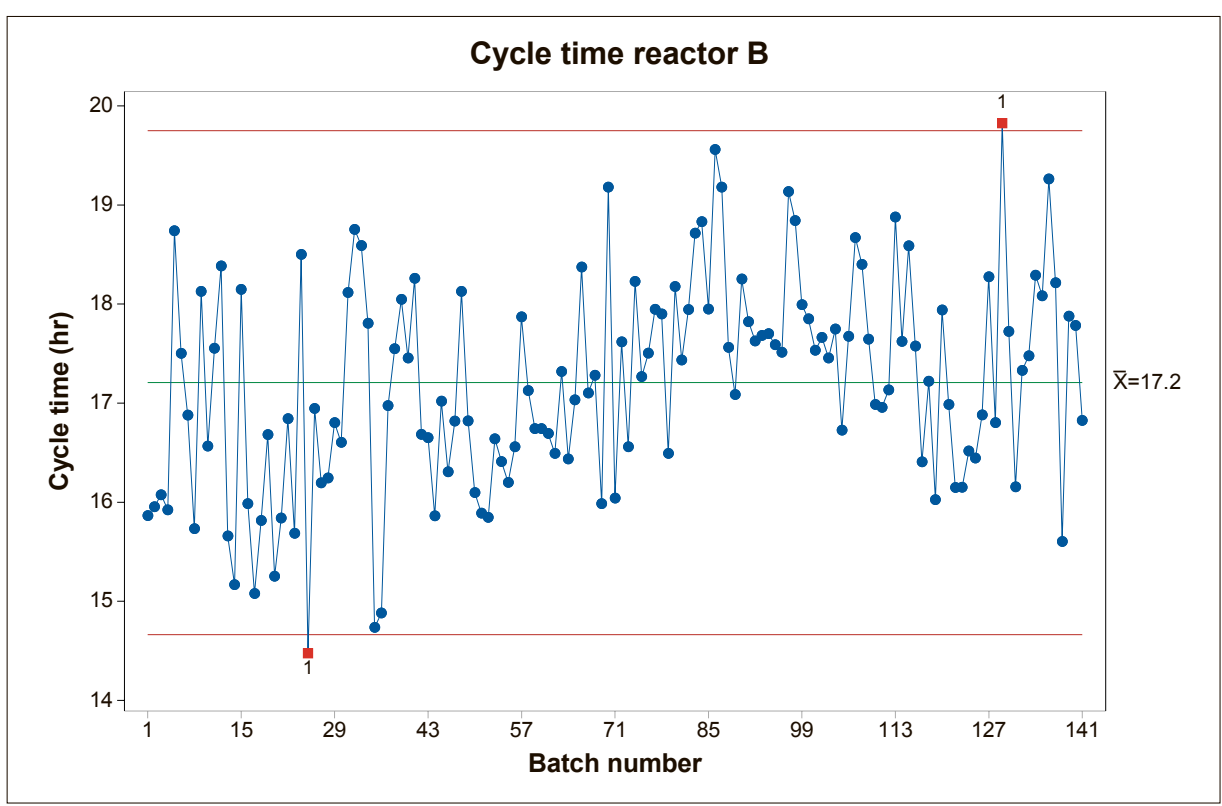

Fig. 2. Representation over time of the primary metric, the cycle time of reactor B; this is the time between the start of one batch, and the start of the next. Shown here is the baseline period, so before improvement measures were taken. The average cycle time was $17.2 \mathrm{~h}$, the standard deviation was $1 \mathrm{~h}$. Based on the variance the upper and lower control limit are calculated, and shown as red lines. A stable process should not come outside these boundaries; in the case it did happen occasionally, one more reason to study the variability.

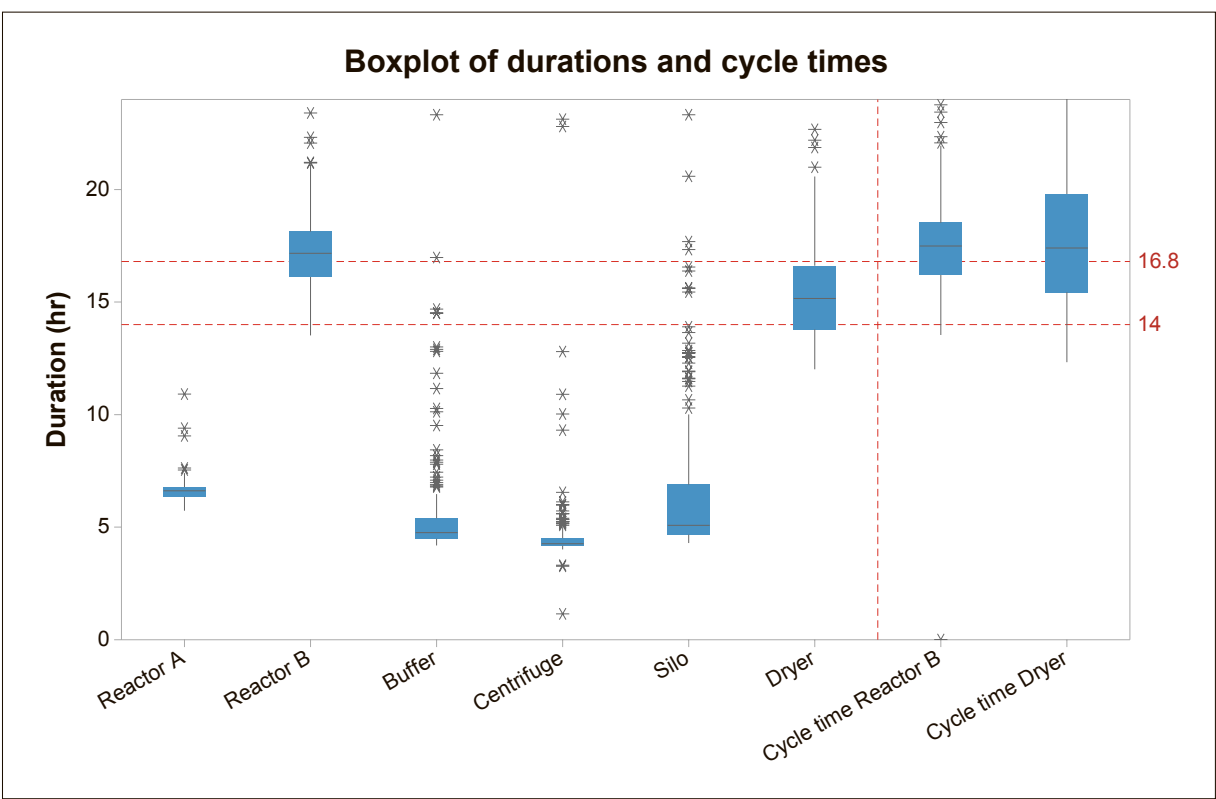

Fig. 3. Duration of the unit operations before improvements have been made. The two boxes to the right denote the cycle times, based on reactor $\mathrm{B}$ and on the dryer. The blue boxes denote the middle $50 \%$ of the data, i.e. all the data between the lowest $25 \%$, and highest $25 \%$. The line in the middle of the box denotes the median ('middle value'), the vertical lines the lowest and highest $25 \%$ of the data. Data points which lie significantly outside the range are denoted with an asterisk, they can be considered as outliers if there are only a few. If a lot of these points are available the data is not normally distributed; which is commonly the case with unit operations that do not take long themselves, but have high values if a delay is present. The buffer vessel and silo are examples of such unit operations. The upper red line denotes the cycle time of the data in the last years. The lower red line, at $14 \mathrm{~h}$, denotes the target of the project. For that clearly the duration of reactor $B$ and the dryer must be decreased. 
and that time data are available to quantify their duration. Some of these process steps were very short, and therefore not relevant for this project, while it is hardly possible to save time with these steps. Two very long steps can be seen in Fig. 4, the transfer of reaction medium from reactor A to B, and the Heat_1 step. The former will be discussed later, the latter could be significantly shortened, by increasing the amount of steam supplied to the reactor jacket. This seems a very easy measure to decrease the duration, but it also shows the power of such a quantitative analysis of the process duration. By quantifying the duration, it became clear that this is an important step to optimize, before it was never in focus. Obviously, the boundaries of the process conditions have been considered, with increasing heat input overheating had to be avoided.

Several shorter process steps were studied as well. There it was observed that several process steps were, for historical reasons, performed sequentially, although they could be done in parallel as well. The plant was built end of the 1980s, at a time when the process control system could only perform tasks sequentially. Later it was migrated to a modern process control system, without any process adaptations. During the current project several steps, e.g. emptying two different vessels, or evacuating and heating, have been programmed in parallel, saving a significant amount of time. This fits very well to the concept of Lean, where steps which do not add value should not take up precious time; by parallelization of these steps parts of this 'waste' was removed.

Apart from investigating the average (or median) duration, the variation is also worth considering. Apparently, the steps with a large variation can be short, but for some reason they were sometimes much longer, meaning there could be improvement potential in removing the long durations. In Fig. 4 the stars denote outliers (here defined as points significantly deviating from the normal distribution). When only a few of these points are present, they are not worth considering, since this graph consists of 170 batches. When the box (representing the middle $50 \%$ of the data) is large, or a lot of 'outliers' are present, it is worth taking a closer look.

The step Transfer from reactor A to $B$ had a large variation, and was the second longest on average. This step consists of waiting until reactor $\mathrm{A}$ is finished and ready for transfer, and the transfer of the reaction medium. The reaction medium of reactor $\mathrm{A}$ is not allowed to stand too long for quality reasons; therefore, the timing should be such that reactor $\mathrm{B}$ is always ready to receive if reactor $\mathrm{A}$ has finished. As a result, there will always be a waiting

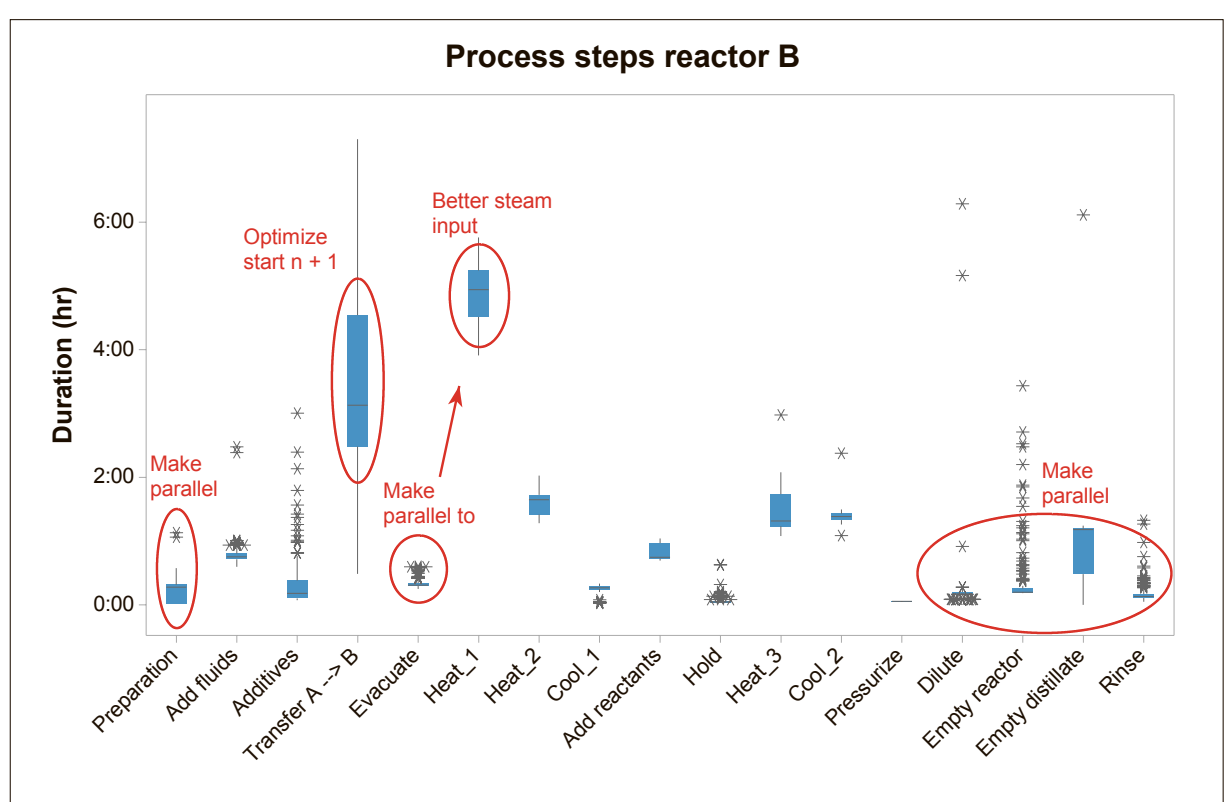

Fig. 4. Duration of the individual process steps of reactor B, consisting of data from 170 batches. Several improvement ideas are depicted here. Parallelization of several process steps will decrease the overall process duration, as well as an increased steam input. The transfer time, which is the time of the transfer itself, including waiting time on reactor A has the largest potential.

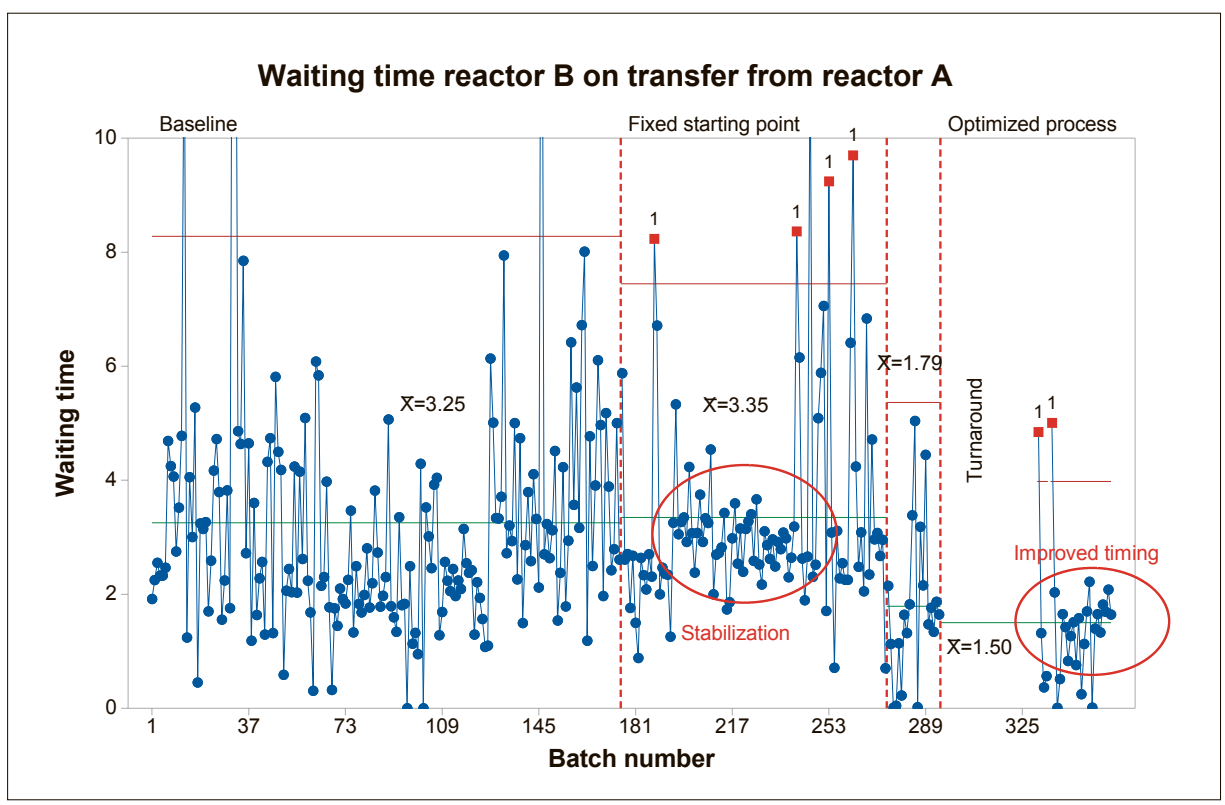

Fig. 5. Waiting time of reactor $B$ on material from reactor $A$. The first time frame, the baseline, is before measures to improve this step have been taken. They show a large variation. By fixing the starting point of reactor $A$ the waiting time could be stabilized significantly. Afterwards the starting point of reactor $A$ was shifted to an earlier moment, additionally several programming changes have been made during the turnaround to decrease the variation in reactor $A$ as well as in reactor $\mathrm{B}$, to make the timing more predictable. With these measures the waiting time was decreased from more than $3 \mathrm{~h}$ to $1.5 \mathrm{~h}$, directly resulting in a shorter cycle time and therefore higher production rate.

time included in this step, goal should be to keep it as short as possible. The waiting time (without transfer) is depicted in Fig. 5 , with the left part being batches before measures were implemented to reduce the waiting time. Large variations can be observed, from more than 8 hours until (almost) zero. Without reducing the variance, it won't be possible to decrease the waiting time, since there is a high risk that reaction medium has to wait too long in reactor A. To explain the large variance the duration of both reactor $\mathrm{A}$ and reactor $\mathrm{B}$ have to be considered. Reactor B is the bottleneck step of the process, it should run continuously. Reactor A only has to be operated part of the time. However, a batch in A should be started before the previous batch in $\mathrm{B}$ is finished, in order to be finished in time. Since delays can happen in reactor B 
afterwards, reactor A was often started relatively late to avoid risks. Until Batch 176 it was not defined formally when it has to be started; the operator could decide himself, based on what he expected on delays and the time both reactors would take. The experience showed that they often stayed on the safe side (starting A later), resulting in a long waiting time. The first measure therefore was giving the operators a clear starting point; unless a critical deviation was to be expected, reactor A should be started at a certain point, defined based on the time data of this project. This resulted in much less (short term) variations, as can be seen in Fig. 5, although the average stayed equal. This paved the way for an earlier start of reactor $\mathrm{A}$, and therefore a shorter waiting time. The variation in starting point was one of the variations, but not the only one. During the following turnaround several measures were taken to decrease the variance. One example was to program an alarm on the transfer rate from $\mathrm{A}$ to $\mathrm{B}$ when the flow rate becomes low, thus the filter can be changed at an appropriate time, and not when it is already clogged. Another variation which plays an important role, but on the long term, is the temperature of cooling water. In Grenzach cooling water is taken from the Rhine; the temperature difference between winter and summer directly corresponds to shorter and longer cooling times of certain process steps. For the starting time of reactor $A$ this means that this difference needs to be taken into account, or a technical solution should be implemented to decrease the effect. In this case the former solution was chosen, since reactor $\mathrm{A}$ is not a bottleneck.

Using these measures, the waiting time of reactor B was more than halved, leading to a decrease in batch duration of approximately 1.5 hours, with potential for more.

\section{Dryer}

The average duration of the dryer was lower than that of reactor B. However, significant variation was present. As a result, sometimes the overall process was slowed down by long dryer durations. Additionally, if the duration of reactor $\mathrm{B}$ is decreased, the dryer will become the bottleneck if no improvements will be made. Therefore, the procedure and performance of the dryer were analyzed as well. This dryer is filled with the solid material, which is separated from the mother liquor in the preceding centrifuge. The pressure is decreased, and the temperature is slowly increased. When reaching a certain temperature, a sample is taken, to check the moisture content of the powder. If this value is within the specifications, the dryer will be cooled down, and the material can be released from the dryer. A close observation of the moisture analysis showed that only in exceptional cases (e.g. with technical problems) the sample did not meet the specification, all other samples were ok. At a defined temperature and pressure, providing sufficient mixing in the dryer, the vapor pressure and therefore moisture content is fixed and therefore moisture specification should also be met. The analysis result is important for the final specification and release of the product; this is a mandatory sample. However, it was agreed that the sample will be taken and analyzed only after the cooling procedure started. This could result in a significant time loss, if the moisture content would be too high and the dryer would need to be heated up again. However, as the analysis of the sample takes half an hour, in which the temperature increases further, the decrease of the duration every drying cycle will easily compensate the exceptional case of a sample not meeting specifications. Additional effort was made to identify at which temperature the material is dry enough, i.e. meets specifications. A simple sample program at different temperatures showed that the end temperature could be decreased significantly without violating the specifications. In combination with the sampling after starting the cooling, the drying time could be decreased by almost three hours, without any investments in equipment and without compromising on quality and 'first time right' ratio.

\section{Control Phase (C)}

In the last phase of DMAIC, the control phase, it must be proven whether the primary metric has improved over a longer time, and whether this change is statistically significant. If that is the case, it also needs to be checked if there is no negative influence on other important parameters (secondary metric(s)). The financial analysis will prove, in hindsight, whether the benefit was worth the effort and possible investments. Finally, measures need to be taken to prevent that the improvements fade away, after the attention of the improvement project is gone.

The combination of different measures in the different unit operations, of which the most important ones were described before, led to a decrease in cycle time of the process (Fig. 6). The overall cycle time thereby was decreased by more than 3 hours, which corresponds to an overall production rate increase of more than $20 \%$. This was achieved without any investments in new equipment or technical changes to current equipment. The changes made were only in the procedure, order and programming of the process. Therefore, in this case it is particularly important to continue to monitor the performance. A dashboard has been made in Excel, where the operators can see the duration for every batch in every unit operation. If it is much longer than normal it gets a different coloring, and they can comment on the cause, if known. This tool ensures that the most important performance killers are recorded, and can be investigated, but it will also show if for some reason the new procedures are not followed anymore, or if some steps are gradually getting longer.

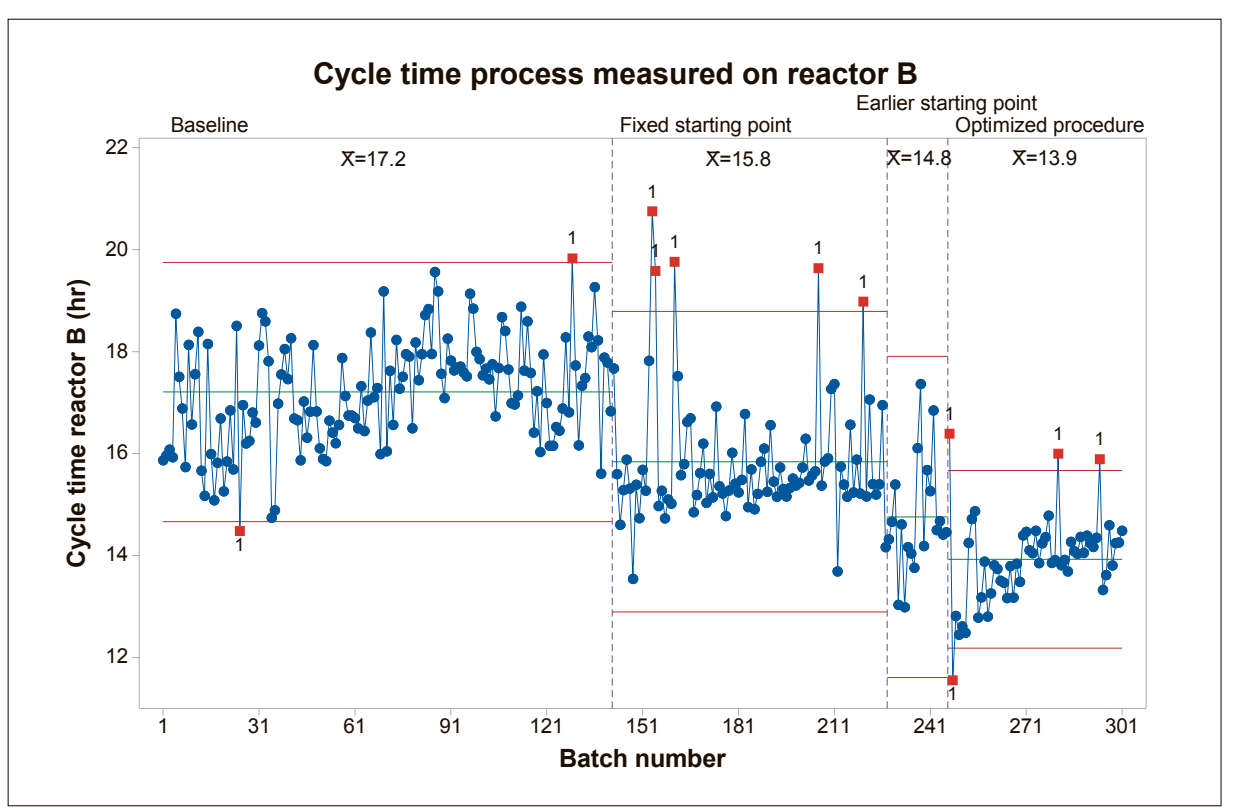

Fig. 6. Cycle time of process, before changes (baseline), after fixing the starting point and after all the project measures were taken (optimized procedure). During the last period 2 batches were much longer than the rest (depicted in red), due to technical problems. The rest of the data only show low variation, the process is more stable than before. The average cycle time was reduced by more than $3 \mathrm{~h}$ (statistically significant), leading to an increased production rate of more than $20 \%$, without any investments in new equipment or technical adaptation of the current plant. All improvements have been made by changing procedures, and the subsequent programming. 


\section{Conclusions}

This paper showed an example of a Lean Six Sigma project performed at DSM, for a typical, though relatively small, batch process. The effort which has been put in this improvement project was substantial in manpower, but without any investment this process has an increased output of more than $20 \%$. The invested work was therefore definitely worth it. The methodology as shown here is applicable to a lot of processes, not only batch processes, but also continuous processes. Within DSM the Lean Six Sigma methodology is therefore applied more and more often, as part of our continuous improvement culture.

\section{Acknowledgements}

The project described here was, like all Lean Six Sigma projects, a team effort. I would therefore like to thank all the people involved, especially the core project team: Monika Knopp, Christina Smuda, Stefan Kornmayer, Hermann-Josef Hilgers and Henrik Leimeister.

Received: January 24, 2018
[1] J.P. Womack; D.T. Jones; D. Roos, 'The Machine That Changed the World', HarperPaperBacks, ISBN-10:0060974176, 1990.

[2] G. Tennant, 'Six Sigma: SPC and TQM in Manufacturing and Services', Routledge, ISBN-10: 0566083744, 2001.

[3] Q. Brook, 'Lean Six Sigma and Minitab', 4th edn., OPEX Resources Ltd., ISBN-10: 09548138X, 2014 